\title{
Caracterização de biocerâmica de fosfatos de cálcio microestruturada em diferentes composições em ovinos ${ }^{1}$
}

\author{
Ademar L. Dallabrida ${ }^{2 *}$, Nelson H.A. Camargo ${ }^{4}$, Aury N. Moraes ${ }^{3}$, Aldo Gava ${ }^{3}$, \\ Gisele M.L. Dalmônico ${ }^{4}$, Bruna D. Costa ${ }^{2}$ e Nilson Oleskovicz ${ }^{3}$
}

\begin{abstract}
Dallabrida A.L., Camargo N.H.A., Moraes A.N., Gava A., Dalmônico G.M.L., Costa B.D. \& Oleskovicz N. 2018. [Bioceramic characterization of calcium phosphates microstructured in different composition in sheep.] Caracterização de biocerâmicas de fosfatos de cálcio microestruturada em diferentes composições em ovinos. Pesquisa Veterinária Brasileira 38(7):1327-1336. Departamento de Medicina Veterinária, Centro de Ciências Agroveterinárias, Universidade do Estado de Santa Catarina, Av. Luis de Camões 2090, Conta Dinheiro, Lages, SC 88520-000, Brazil. E-mail: ademar.dallabrida@udesc.br

Microporous bioceramics of calcium phosphate and biphasic hydroxyapatite and tricalcium phosphate beta (HA/TCP- $\beta$ ) in the form of microporous granules biomaterials are research subjects and stand out as bone substitutes in biomedical application. The biphasic bioceramics are biocompatible, bioactive, osteoinductive and promote osseointegration when applied in vivo or through simulated. Another aspect of such differential solubility bioceramics is associated with the capacity that these biomaterials exhibit when applied in biological environment, enabling the gradual release of calcium and phosphate ions to the biological environment they are absorbable and promote neogenesis of new bone tissue. The biphasic bioceramics of calcium phosphate also have promising applications in traumatology in the repair of injured bone and controlled release of drugs in the bone structure treatments. The performance of these biomaterials as bone substitutes and controlled release of drugs, are associated, their physical, chemical, morphological and surface. The aim of this study was to make morphological, microstructural of biomaterials by the technique of scanning electron microscopy (SEM), physics with X-ray diffraction (XRD) and Arthur method for determination of open porosity. The theoretical density of biphasic biomaterials was determined by theoretical method of biphasic concentrations. Finally, we conducted evaluation of osteogenesis and osseointegration behavior of different biomaterials of calcium phosphates in vivo tests on sheep. In vivo tests were performed on tibias of sheep up to of 03 months. The biomaterials implanted were hydroxyapatite (HA), tricalcium phosphate- $\beta$ ( $\beta$-TCP) and biphasic compositions as HA/TCP- $\beta$ in rates: $80 / 20,20 / 80,70 / 30$ and 30/70. Eight crossbred Texel sheep, with 12 months of age and average weight of $30 \mathrm{~kg}( \pm 5 \mathrm{~kg})$ were used, in which were produced three bone defects in the tibia, four of these defects were filled with biomaterials, and two by bone fragments (autograft), as control group. The animals were euthanized at 03 months after implantation of biomaterials. After euthanasia, the tibias were collected for evaluation using the scanning electron microscopy technique (SEM). The results show that the microporous biomaterial granules are formed by an irregular morphology with grain size between $200 \mu \mathrm{m}$ and $500 \mu \mathrm{m}$, another finding was microporous interconnected microstructure of biomaterials. The result showed that the open porosity of the biomaterials exhibit porosity greater than
\end{abstract}

\footnotetext{
${ }^{1}$ Recebido em 5 de junho de 2017.

Aceito para publicação em 20 de junho de 2017.

2 Programa de Pós-Graduação em Ciência Animal, Centro de Ciências Agroveterinárias (CAV), Universidade do Estado de Santa Catarina (UDESC), Av. Luiz de Camões 2090, Conta Dinheiro, Lages, SC 88520-000, Brasil. E-mails: bruna_ditzel@yahoo.com.br; *Autor para correspondência: ademar.dallabrida@udesc.br

${ }^{3}$ Departamento de Medicina Veterinária, Centro de Ciências Agroveterinárias (CAV), Universidade do Estado de Santa Catarina (UDESC), Av. Luis de Camões
}

2090, Conta Dinheiro, Lages, SC 88520-000. E-mails: aury.moraes@cav.udesc.br, noleskovicz@yahoo.com.br, aldo.gava@cav.udesc.br

${ }^{4}$ Departamento de Engenharia Mecânica, Centro de Ciências Tecnológicas (CCT), Universidade do Estado de Santa Catarina (UDESC), Rua Paulo Malschitzki s/n, Campus Universitário Prof. Avelino Marcante, Zona Industrial Norte, Joinville, SC 89219-710, Brasil. E-mails: nelson.camargo@udesc.br, gidalmonico@hotmail.com 
$68 \%$. The theoretical density was relatively similar between the granulates biomaterials of calcium phosphates and suggest good capacity of osteogenesis for all biomaterials, with the biphasic absorption of the biomaterial introduced 20/80 and more rapid bone formation when compared with other biomaterials evaluated in study.

INDEX TERMS: Bioceramic characterization, calcium phosphate microstructured, sheep, biomaterial, hydroxyapatite, beta-tricalcium phosphate, bone formation, surgery.

RESUMO.- As biocerâmicas microporosas de fosfatos de cálcio e bifásicas de hidroxiapatita e fosfato tricálcico beta (HA/TCP- $\beta$ ) na forma de biomateriais granulados microporosos, são temas de pesquisas e se destacam como substitutos ósseos em aplicações biomédicas. As biocerâmicas bifásicas são biocompatíveis, bioativas, osteoindutoras e promovem a osteointegração, quando aplicados in vivo ou em meio simulado. Outro ponto diferencial dessas biocerâmicas está associado à capacidade de solubilidade que esses biomateriais apresentam quando aplicados em meio biológico, permitindo a liberação gradual de íons cálcio e fosfatos para o meio biológico, estes são absorvíeis e promovem a neoformação de um novo tecido ósseo. As biocerâmicas bifásicas de fosfatos de cálcio também se apresentam promissores em aplicações traumatológicas na reparação do tecido ósseo traumatizado e na liberação controlada de medicamentos, em tratamentos da estrutura óssea. 0 desempenho desses biomateriais como substitutos ósseos e na liberação controlada de medicamentos, estão associados, as suas características físicas, químicas, morfológicas e de superfície. 0 objetivo desse estudo foi realizar a caracterização morfológica, microestrutural dos biomateriais pela técnica de microscopia eletrônica de varredura (MEV), física com difratometria de raios X (DRX) e método de Arthur para determinação da porosidade aberta. A densidade teórica dos biomateriais bifásicos foi determinada pelo método teórico das concentrações bifásicas. Por fim, se realizou avaliação do comportamento da neoformação óssea e osteointegração dos diferentes biomateriais de fosfatos de cálcio em testes in vivo em ovinos. Os testes in vivo foram realizados em tíbias de ovinos com tempo de implantação de 03 meses. Os biomateriais implantados foram hidroxiapatita (HA), fosfato tricálcico- $\beta$ (TCP- $\beta$ ) e composições bifásicas HA/TCP- $\beta$ nas proporções: $80 / 20$, $20 / 80,70 / 30$ e $30 / 70$. Foram utilizadas 08 ovelhas mestiças Texel, com 12 meses de idade e peso médio de $30 \mathrm{~kg}( \pm 5 \mathrm{~kg})$, nas quais foram produzidos três defeitos ósseos em cada tíbia, sendo que quatro desses defeitos foram preenchidos por biomateriais, e dois por fragmentos ósseos (autoenxerto), grupo controle. Os animais foram eutanasiados aos 03 meses após a implantação dos biomateriais. Após a eutanásia, foram coletadas as tíbias para avaliação com o uso da técnica de microscopia eletrônica de varredura (MEV). Os resultados encontrados mostram que os biomateriais granulados microporosos são formados por uma morfologia irregular com tamanho de grânulos entre 200 $\mu \mathrm{m}$ e $500 \mu \mathrm{m}$, outra constatação foi microestrutura microporosa interconectada dos biomateriais. 0 resultado obtido da porosidade aberta mostrou que os biomateriais apresentam porosidade superior a 68\%. A densidade teórica se apresentou semelhante entre os biomateriais granulados de fosfatos de cálcio e sugerem boa capacidade de neoformação óssea para todos os biomateriais, sendo que o bifásico 20/80 apresentou absorção do biomaterial e neoformação óssea mais rápida quando comparada com os outros biomateriais avaliados neste estudo.
TERMOS DE INDEXAÇÃO: Biocerâmica, fosfato de cálcio microestruturada, ovinos, biomaterial, hidroxiapatita, fosfato tricálcico- $\beta$, neoformação óssea, cirurgia.

\section{INTRODUÇÃo}

As biocerâmicas de fosfatos de cálcio e bifásicas de hidroxiapatita (HA)/fosfato tricálcico beta (TCP- $\beta$ ), são biomateriais biocompatíveis e apresentam similaridade cristalográfica com a apatita dos ossos e dentes e desempenham o papel da osteoindução e da osteointegração quando aplicados in vivo (Gauthier et al. 1998, Yuan et al. 1998, Kurashina et al. 2002, Camargo et al. 2014).

As biocerâmicas de fosfatos de cálcio na forma de biomateriais granulados ou de blocos microporosos se destacam nas pesquisas como biomateriais de reparação de defeitos e de reconstrução do tecido ósseo, como observado por diferentes autores, que realizaram teste in vivo e observaram que esses biomateriais, desempenham a neoformação óssea quando aplicadas in vivo (Livingston et al. 2003, Wang et al. 2007, Ghannati et al. 2012, Tanaka et al. 2012).

Constata-se, que o desempenho dessas biocerâmicas sobre o processo de neoformação óssea, está associado diretamente às características de bioatividade, capacidade de solubilidade, molhabilidade e microestruturas microporosas interconectadas, elevada área superficial de grãos e de microporos (Flautre et al. 2001, Dalmônico 2011, Camargo et al. 2012). Essas características oferecem condições para adesão de células de osteoblastos na superfície do biomaterial granulado bem como, para o interior da microporosidade interconectada através do prolongamento das células de osteoblastos (Costa et al. 2015), para o interior da microporosidade do biomaterial granulado, levando a biomineralização do tecido ósseo (Rosa et al. 2002, Samavedi et al. 2013, Dalmônico 2015). As biocerâmicas de fosfato de cálcio microporosas são fabricadas com uma arquitetura similar à da estrutura do tecido ósseo, formadas por finos grãos, com microporosidade interconectada, que auxiliam no mecanismo de adesão e proliferação celular, com a osteoindução, osteointegração e a formação de um novo tecido ósseo (Dubois et al. 1999, Ramay \& Zhang 2004, Dorozhkin 2012, Levandowski Junior 2014).

O objetivo desse trabalho foi caracterizar diferentes biomateriais de fosfatos de cálcio e comparar o comportamento da neoformação óssea e osteointegração entre as diferentes biocerâmicas de hidroxiapatita (HA), fosfato tricálcico- $\beta$ (TCP- $\beta$ ), e bifásicas: HA/TCP- $\beta$ 80/20, HA/TCP- $\beta$ 20/80, (HA/TCP- $\beta$ 70/30 e HA/TCP- $\beta 30 / 70$, com interesse de aplicações como substitutos ósseos, implantados em defeitos intraósseos realizados experimentalmente em tíbias de ovelhas. Muitas vantagens são pertinentes ao uso da ovelha em detrimento ao cão, como por exemplo aspectos relacionados ao bem-estar animal, padronização dos animais e maior facilidade de transferir os resultados para os seres humanos sem a necessidade de estudos adicionais relacionados as questões de biocompatibilidade (Nuss et al. 2006). 


\section{MATERIAL E MÉTODOS}

Caracterização morfológica e microestrutural dos biomateriais (microscopia eletrônica de varredura - MEV). Os estudos de caracterização morfológica microestrutural dos biomateriais granulados microporos foram realizados com o uso de um microscópio eletrônico de varredura, marca ZIESS, modelo DSM 940A, através do sistema de elétrons secundários (SE).

Para facilitar os trabalhos de caracterização com a técnica de microscopia eletrônica de varredura (MEV), os biomateriais granulados microporosos foram preparados, utilizando "uma porta" amostra na forma de disco em liga de alumínio com superfície lisa, uma fita de carbono dupla face foi fixada na superfície lisa do disco, onde se fixou cuidadosamente o biomaterial granulado a superfície da fita de carbono.

A fim de evitar os efeitos de carga dentro da câmara do microscópio, todos os biomateriais granulados foram submetidos ao processo de metalização por pulverização catódica com deposição de um filme de ouro sobre a superfície do biomaterial. Esta deposição foi realizada através de um metalizador marca Bal-Tec modelo SCD 050 Sputter Coater, sendo os parâmetros de deposição iônica estabelecidos da seguinte forma, temperatura na câmara de metalização de $25^{\circ} \mathrm{C}$, corrente de $40 \mathrm{~mA}$, tensão de $2 \mathrm{kV}$ e tempo de deposição de 140 segundos, fornecendo um filme de ouro na superfície do biomaterial granulado da ordem de $30 \mathrm{~nm}$. Para cada segmento ósseo enviado para as análises de MEV foram realizadas 20 micrografias, totalizando 480 MEVs.

Caracterização da porosidade aberta e densidade teórica dos biomateriais ( $\left(\mathrm{PA}\right.$ e $\mathrm{d}_{\mathrm{th}}$ ). A caracterização da porosidade aberta em porcentagem foi realizada para os diferentes biomateriais granulados microporosos de fosfatos de cálcio. As análises foram realizadas utilizando o método de Arthur (Pennings \& Grellner 1989). Este método consiste em medir o peso dos biomateriais granulados ao ar (Par), depois o peso embebido em xileno (Pxa). 0 xileno penetra nos poros abertos e posteriormente mede-se o peso do biomaterial com xileno depois de imerso em água destilada (Pxe). Após realizar essas medidas se determinou os valores da porosidade aberta (PA) através da equação:

$$
P A=\frac{\left(P_{x a}-P_{a r}\right) \cdot d_{e}}{\left(P_{x a}-P_{x e}\right) \cdot d_{x}} \cdot 100
$$

Onde:

$\mathrm{PA}=$ porosidade aberta $(\%)$;

Par $=$ massa do corpo seco, pesado ao ar $(\mathrm{g})$;

Pxa = massa do corpo com xileno, pesado ao ar (g);

$\mathrm{Pxe}=$ massa do corpo com xileno, pesado imerso em água destilada (g); $\mathrm{de}=$ densidade da água $(\mathrm{g} / \mathrm{cm} 3)$;

$\mathrm{dx}=$ densidade do xileno $(0,8802 \mathrm{~g} / \mathrm{cm} 3)$;

$\mathrm{dh}=$ densidade hidrostática $(\mathrm{g} / \mathrm{cm} 3)$;

$\mathrm{dt}=$ densidade teórica $(\mathrm{g} / \mathrm{cm} 3)$.

A densidade teórica $\left(\mathrm{d}_{\mathrm{th}}\right)$ dos biomateriais granulados microporosos bifásicos foi determinada com o uso do método teóricos de composições bifásicos, desenvolvido por (Eckstein 2016). Esse método envolve a concentração em porcentagem das composições e suas densidades. 0 cálculo é realizado de acordo com a equação:

$$
d_{t h}(A+B)=d A \cdot A+d B \cdot B
$$

Onde:

$\mathrm{d}_{\text {th }}(\mathrm{A}+\mathrm{B})=$ densidade teórica da composição $\mathrm{A}+\mathrm{B}\left(\mathrm{g} / \mathrm{cm}^{3}\right)$;

$\mathrm{dA}$ e $\mathrm{dB}=$ densidade teórica do material $\mathrm{A}$ e $\mathrm{B}$, respectivamente $\left(\mathrm{g} / \mathrm{cm}^{3}\right)$;

$\mathrm{A}$ e $\mathrm{B}=$ fração dos materiais $\mathrm{A}$ e $\mathrm{B}$, presentes na composição, respectivamente.

Caracterização física (DRX). Os estudos de caracterização cristalográfica foram realizados para os diferentes biomateriais granulados microporosos de fosfatos de cálcio. Este trabalho foi realizado com o uso do equipamento de difratometria de raios $\mathrm{X}$, marca SHIMADZU modelo XRD_6000, com anticátodo tubo de cobre. Para obtenção dos difratogramas de raios X, utilizou tensão de $40 \mathrm{kv}$ e intensidade de corrente de $30 \mathrm{~mA}$, sendo o intervalo angular de varredura de $20^{\circ}$ a $67^{\circ}$, com velocidade do goniômetro de $2^{\circ} / \mathrm{min}$ em função de $2 \theta$.

Delineamento experimental. Estudo aprovado pelo Comitê de Ética e de Bem-Estar Animal - CETEA da instituição de origem, protocolo n. 1.70.12. Foram utilizadas oito ovelhas mestiças Texel, hígidas, com peso médio de 28,5 $\pm 7,4 \mathrm{~kg}$ e 12 meses de idade. Previamente à inclusão na pesquisa, todos os animais foram submetidos à avaliação clínica, radiográfica e laboratorial (hemograma, função renal (ureia e creatinina) e função e lesão hepática (aspartato aminotransferase, fosfatase alcalina, proteína sérica total, albumina, globulinas), a fim de excluir animais com afecções que pudessem comprometer a pesquisa. Os animais selecionados foram alocados em baias no setor de grandes animais do Hospital Veterinário para um período de adaptação de no mínimo, 15 dias, recebendo medicação antiparasitária, alimentação à base de ração comercial para ovinos com $18 \%$ de proteína e silagem de milho, além de água ad libitum.

0 acesso à tíbia foi realizado conforme técnica descrita por Costa et al. (2015). Uma vez realizada a exposição da diáfise tibial em sua face medial, produziu-se três defeitos ósseos na face medial da diáfise tibial de cada uma das tíbias. Dois destes defeitos foram produzidos com auxílio de uma broca odontológica de $6 \mathrm{~mm}\left(\right.$ Neodent $^{\circledR}$, Curitiba, PR), os quais foram preenchidos por biomateriais. Os defeitos produzidos para estudo controle, foram fabricados com auxílio de uma trefina de $6 \mathrm{~mm}$, a fim de possibilitar a remoção do fragmento ósseo a ser reimplantado. 0 defeito preenchido com o fragmento ósseo estava localizado sempre na posição proximal da diáfise tibial (Fig.1). Durante a realização dos defeitos, o local de perfuração, foi constantemente irrigado com solução salina estéril resfriada para auxiliar na prevenção de lesões térmicas. 0 cirurgião-auxiliar segurava o membro nas duas extremidades da tíbia, a fim de exercer pressão contrária ao sentido de perfuração, evitando-se a atuação das forças de envergamento.

Os defeitos produzidos tiveram o seu leito preenchido com seis diferentes tipos de biomateriais (Departamento de Engenharia Mecânica, CCT, UDESC/Joinville/SC), hidroxiapatita (HA, n=8), fosfato tricálcico- $\beta$ (TCP- $\beta$, n=8), HA/TCP- $\beta$ 80/20, n=4) HA/TCP- $\beta 20 / 80$, $n=4)$ HA/TCP- $\beta$ 70/30, n=4) HA/TCP- $\beta 30 / 70, n=4$ ); os dois defeitos restantes foram preenchidos pelos fragmentos ósseos removidos com trefina, do próprio animal, correspondendo ao grupo controle (Fig.1).

Para melhor manipulação e implantação nos respectivos orifícios, o biomaterial granulado foi depositado em uma cuba estéril e em seguida, hidratado com sangue arterial, proveniente do próprio animal, conforme descrito por Regalin (2014). Esta mistura deu origem a uma massa viscosa, que facilitou o completo preenchimento dos defeitos ósseos, minimizando a perda de grânulos do biomaterial.

Finalizada a colocação dos biomateriais, bem como do fragmento ósseo, procedeu-se à sutura das camadas teciduais. A reaproximação dos planos anatômicos foi realizada mediante sutura do periósteo e subcutâneo com fio de náilon nํ-2-0 em padrão contínuo simples. 

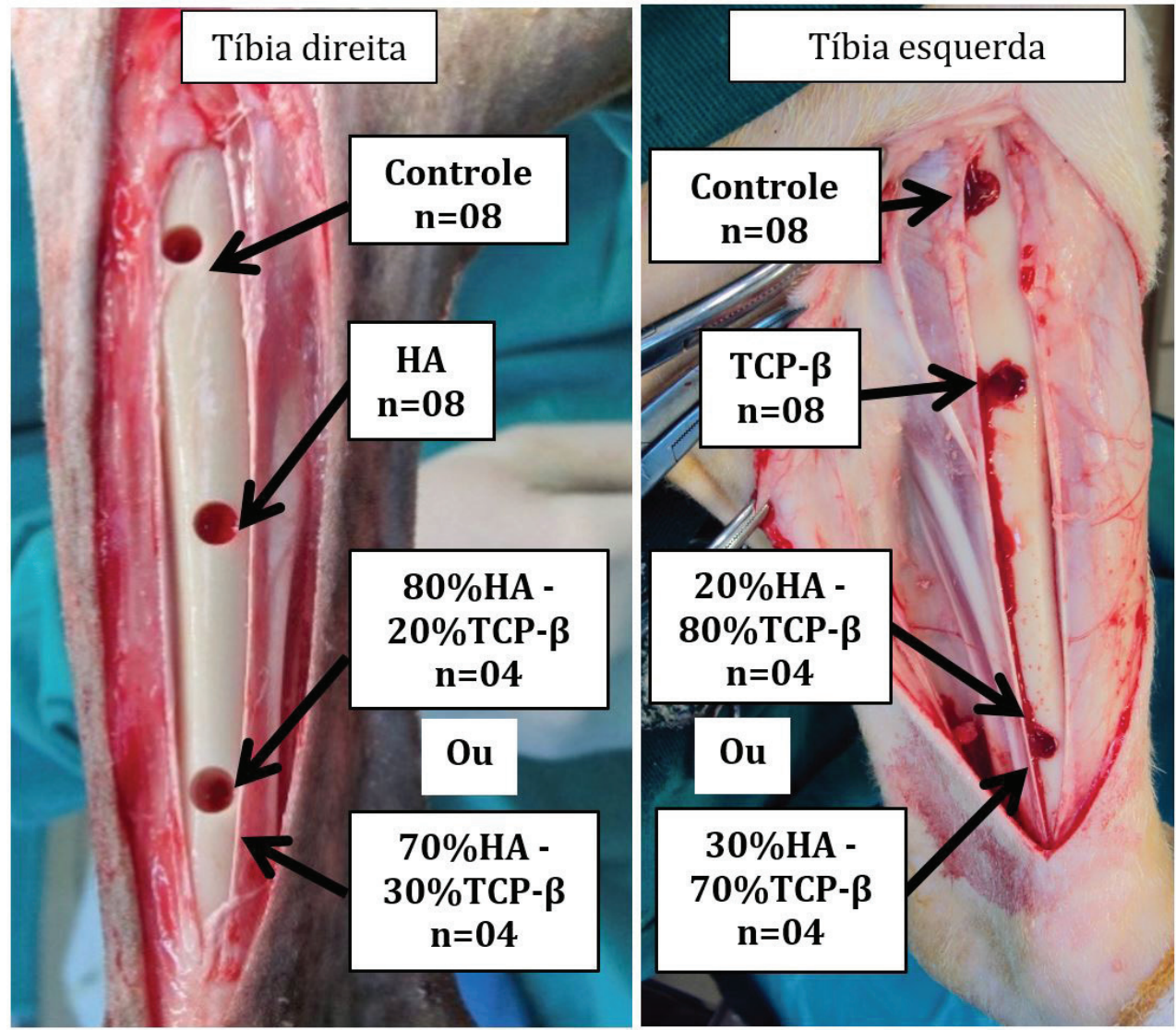

Fig.1. Orifícios de 6 milímetros de diâmetro realizados nas tíbias direita e esquerda de ovelhas, para posterior preenchimento com seus respectivos biomateriais e o grupo controle, sendo "n" o número de orifícios preenchidos com cada tratamento, totalizando 48 orifícios.

Para a dermorrafia foi utilizado fio de náilon 2-0 em pontos isolados simples (Fig.2).

Decorridos 90 dias da implantação dos biomateriais, os animais foram eutanasiados, seguindo-se as normas do Conselho Nacional de Controle de Experimentação animal (CONCEA 2013). Os ossos coletados foram adequadamente identificados e acondicionados em frascos contendo formol 10\%, pelo período de 30 dias. Após a fixação pelo formol a $10 \%$ os ossos foram divididos em três fragmentos, correspondentes a cada segmento implantado com o biomaterial e o autoenxerto e foram encaminhados para análise por microscopia eletrônica de varredura (MEV). As amostras foram primeiramente desidratadas em álcool etílico $70 \%$, por $48 \mathrm{~h}$. Em seguida, foram desidratadas em álcool etílico absoluto $100 \%$ por $56 \mathrm{~h}$, depois colocadas em Xilol por $2 \mathrm{~h}$, para realização da limpeza das amostras. Após, foram imersas novamente em álcool absoluto $100 \%$ por mais $24 \mathrm{~h}$, para eliminação do Xilol. As amostras obtidas foram fraturadas na região onde houve o enchimento ósseo, para posterior preparação para os estudos de caracterização por microscopia eletrônica de varredura (MEV).

\section{RESULTADOS}

\section{Caracterização dos biomateriais (MEV)}

Os resultados obtidos da caracterização morfológica e microestrutural dos biomateriais granulados de fosfatos de cálcio obtidos pela microscopia eletrônica de varredura mostraram que os biomateriais são formados por uma morfologia granular com formas irregulares e com tamanhos de grânulos entre $200 \mu \mathrm{m} \leq \mathrm{d} \leq 500 \mu \mathrm{m}$ (Fig.3A). A Figura 3B mostra o resultado obtido sobre a superfície do biomaterial granulado, revelando a microestrutura microporosa interconectada formada por finos grãos.

\section{Caracterização da porosidade aberta (PA) e densidade teórica $\left(d_{t h}\right)$}

Os resultados da porosidade aberta encontrados para os diferentes biomateriais mostraram valores semelhantes entre os biomateriais, constatando valor superior da porosidade aberta para o biomaterial de hidroxiapatita, conforme ilustrados no Quadro 1. Os resultados obtidos da densidade teórica para os diferentes biomateriais granulados microporos, revelou valores bastante próximos entre os biomateriais granulados, constatando valor superior para o biomaterial bifásico $80 \% / 20 \%$, conforme mostrado no Quadro 1. Esses resultados se associados aos obtidos pela microscopia eletrônica de varredura, sugerem que os biomateriais são semelhantes entre si e revelam característica físicas e promissoras, para uso em aplicações biomédicas, como biomateriais de substituição óssea na reparação de defeitos e da reconstrução do tecido ósseo. Estas microestruturas microporosas interconectadas formada por finos grãos, oferecerem melhores condições de molhabilidade, capilaridade, oxigenação, adesão e proliferação 

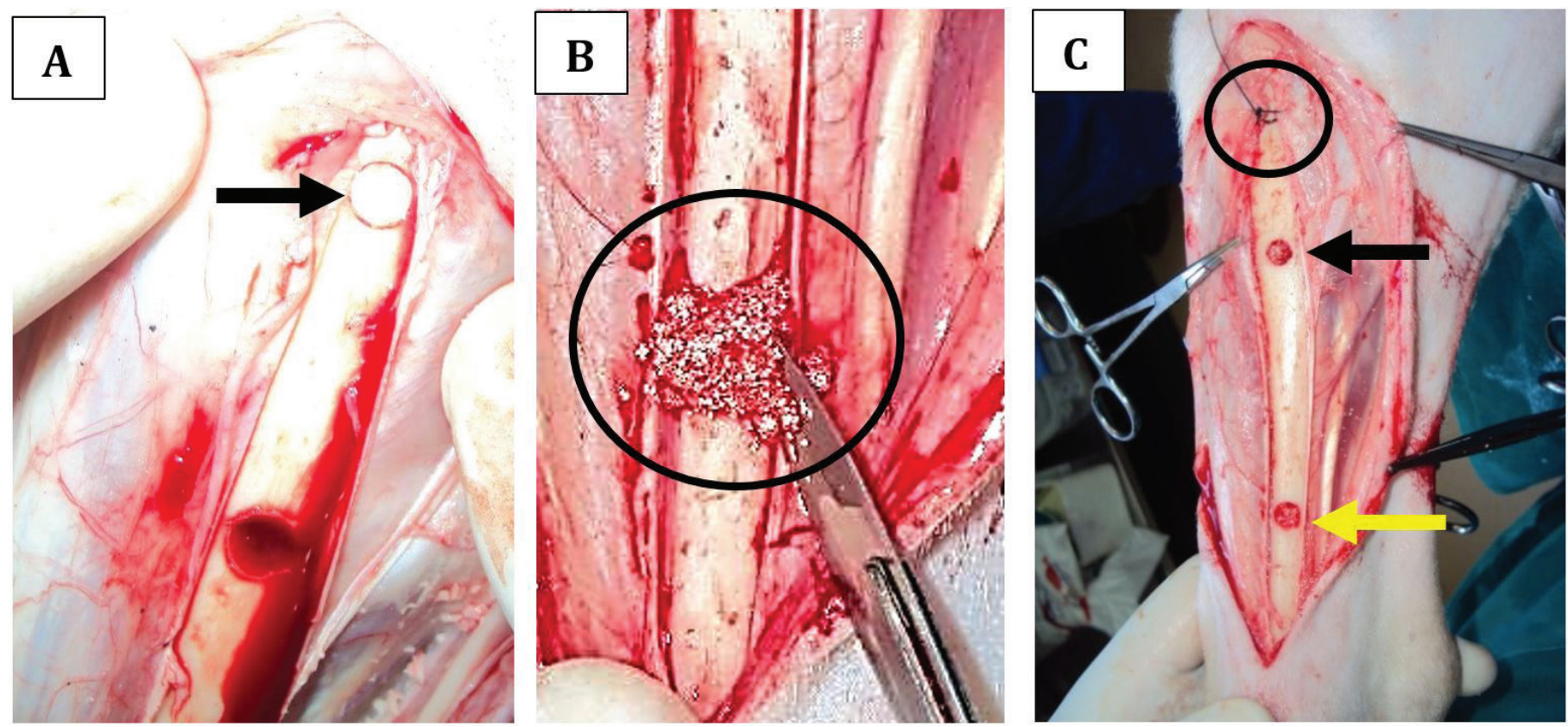

Fig.2. (A) Acesso cirúrgico demonstrando o orifício proximal (seta) com o tratamento controle. (B) Momento em que um dos orifícios está sendo preenchido com biomaterial (círculo). Notar que o mesmo se apresenta hidratado com sangue deixando-o com consistência pastosa. (C) Momento final em que os orifícios estão preenchidos com seus respectivos biomateriais (setas). Início da sutura do periósteo (círculo preto) o tratamento controle se encontra abaixo deste ponto.
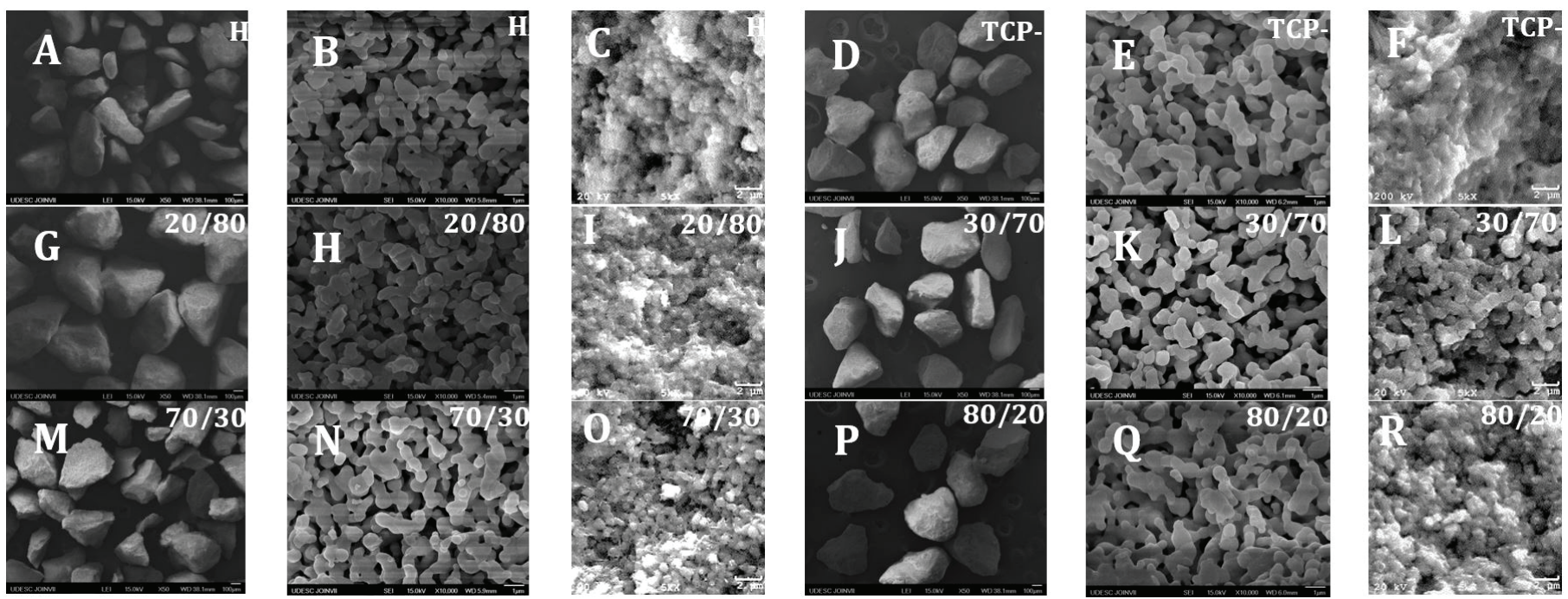

Fig.3. Morfologia e microestrutura dos biomateriais granulados microporosos de hidroxiapatita (HA), fosfato tricálcico beta (TCP- $\beta$ ) e os bifásicos HA/TCP- $\beta$ 80/20, HA/TCP- $\beta$ 20/80, HA/TCP- $\beta$ 70/30 e HA/TCP- $\beta$ 30/70. As figuras (A, B, D, E, G, H, J, K, M, N, P e Q) revelam as morfologias dos biomateriais granulados com formas irregulares e tamanho de grânulos entre $200 \mu \mathrm{m} \leq \mathrm{d} \leq 500 \mu \mathrm{m}$, antes da implantação nos orifícios elaborados nas tíbias de ovelhas. As figuras (C, F, I, L, $\mathbf{O}$ e R) demonstram as microestruturas microporosas interconectadas formadas por finos grãos do biomaterial granulado e osso neoformado após 3 meses de implantação em tíbias de ovelhas.

celular na superfície de grãos e de microporos, promovendo a osteoindução, a osteointegração e a neoformação de tecido ósseo precoce, caso já observado por diferentes autores (Gauthier et al. 1998, Kurashina et al. 2002, Livingston et al. 2003, Costa-Rodrigues et al. 2012, Ghannati et al. 2012, Levandowski Junior 2014, Dalmônico 2015).

\section{Caracterização por difratometria de raios-X (DRX)}

0 resultado obtido pela difratometria de raios $\mathrm{X}$, sobre o biomaterial de hidroxiapatita (HA) revelou em seus difratogramas de raios $\mathrm{X}$ os picos representativos da fase hidroxiapatita (HA) estequiometria na composição $\left(\mathrm{Ca}_{10}\left(\mathrm{PO}_{4}\right)_{6}(\mathrm{OH})_{2}\right.$ com plano principal de difração (211). 
0 resultado obtido sobre o biomaterial TCP- $\beta$ revelou os picos representativos da fase fosfato tricálcico- $\beta$ na composição $\left(\mathrm{Ca}_{3}\left(\mathrm{PO}_{4}\right)_{2}\right.$ com plano principal de difração (021). Os resultados obtidos sobre os biomateriais bifásicos

Quadro 1. Resultados da porosidade aberta (PA) e densidade teórica $\left(d_{t h}\right)$ obtida sobre os biomateriais granulados microporosos

\begin{tabular}{ccc}
\hline Biomaterial granulado & PA (\%) & $\mathrm{d}_{\mathrm{th}}\left(\mathrm{g} / \mathrm{cm}^{3}\right)$ \\
\hline HA & $77,64 \pm 1,67$ & $3,16 \pm 0,001$ \\
TCP- $\beta$ & $73,57 \pm 0,82$ & $3,07 \pm 0,001$ \\
HA/TCP- $\beta(20 / 80)$ & $69,67 \pm 0,44$ & $3,12 \pm 0,004$ \\
HA/TCP- $\beta(80 / 20$ & $75,87 \pm 0,65$ & $3,14 \pm 0,001$ \\
HA/TCP- $\beta(30 / 70)$ & $71,43 \pm 0,26$ & $3,10 \pm 0,001$ \\
HA/TCP- $\beta(70 / 30)$ & $74,29 \pm 0,21$ & $3,13 \pm 0,001$
\end{tabular}

HA/TCP- $\beta$ 20/80, 80/20, 30/70 e 70/30 revelaram em seus difratogramas de raios $\mathrm{X}$, os picos representativos das fases hidroxiapatita e fosfato de tricálcico- $\beta$ nas composições $\left(\mathrm{Ca}_{10}\left(\mathrm{PO}_{4}\right)_{6}(\mathrm{OH})_{2}\right.$ e $\left(\mathrm{Ca}_{3}\left(\mathrm{PO}_{4}\right)_{2}\right.$ respectivamente, conforme observado nos difratogramas de raios X (Fig.4). Outra constatação para os biomateriais bifásico foi o aumento da intensidade dos picos principais (211) HA e (021) para o TCP- $\beta$, conforme se alternou o aumento da concentração destas fases para cada composição de biomateriais bifásicos (Fig.4).

\section{Microscopia eletrônica de varredura dos tecidos biológicos}

De um modo geral, em todos os materiais, os grânulos dos enxertos foram bem integrados com vários graus de remodelação ativa ocorrendo aos 3 meses. Todos os biomateriais apresentaram graus variados de absorção ao longo do período de avaliação, sendo que os mais promissores foram HA/TCP- $\beta$
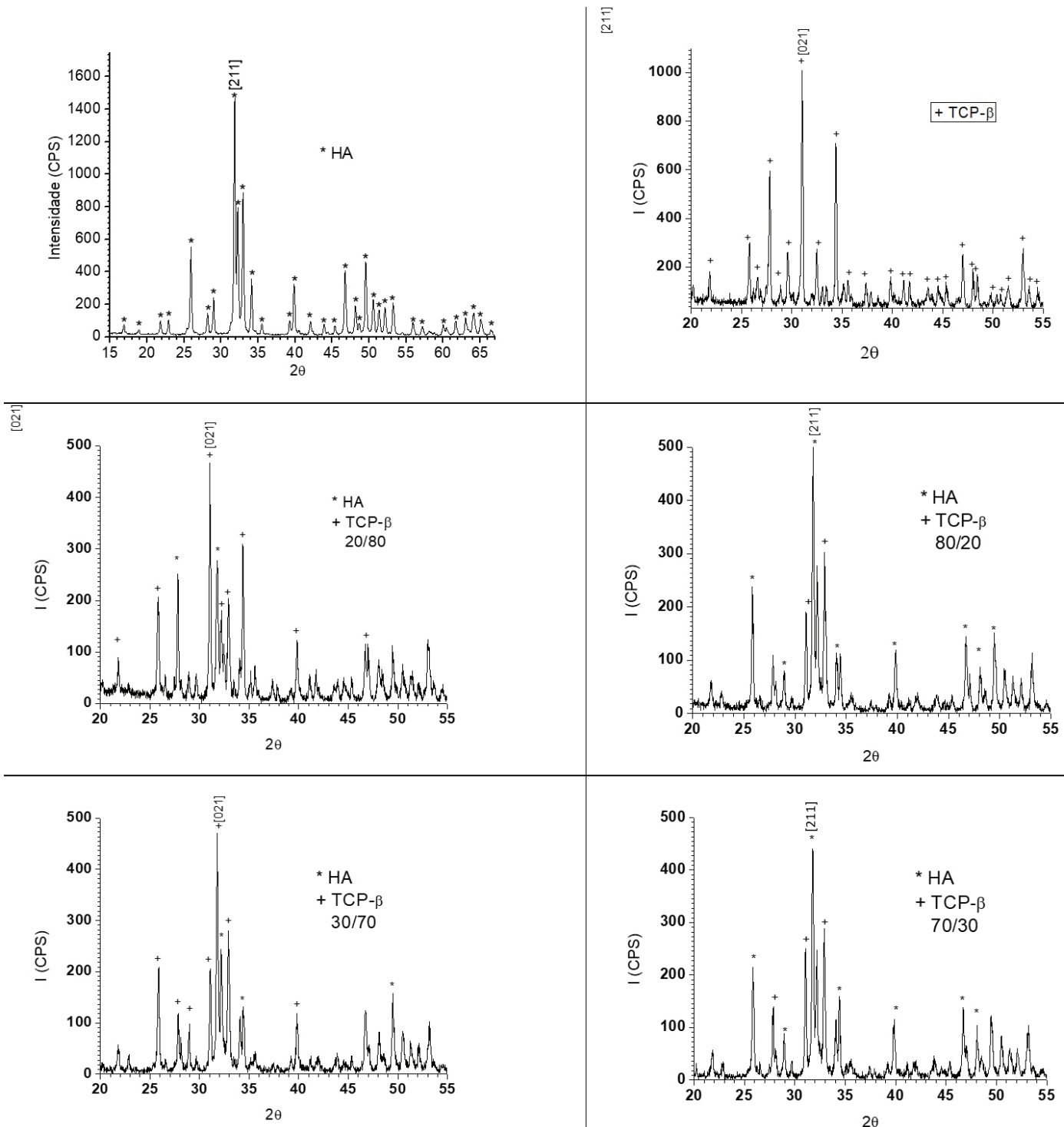

Fig.4. Difratogramas de Raios X (DRX) obtidos sobre os biomateriais HA, TCP- $\beta$ e os bifásicos HA/TCP- $\beta$ (20/80), HA/TCP $\beta$ (80/20), HA/TCP - $\beta(30 / 70)$ e HA/TCP $\beta(70 / 30)$. 
20/80 e o HA/TCP- $\beta 30 / 70$, seguidos pelo TCP- $\beta$, HA/TCP- $\beta$ $80 / 20$, HA/TCP- $\beta$ 70/30 e a HA, respectivamente. A análise das MEVs comparando-se os biomateriais entre si, dentro de cada grupo, permite relacionar a extensão de região cortical onde o biomaterial foi absorvido bem como a extensão de osso neoformado (Fig.5).
Foi possível observar também, através das avaliações das MEVs, que todos os biomateriais aos 3 meses revelaram grande quantidade de osteoblastos sobre as suas superfícies (Fig.6A e B), esses osteoblastos apresentaram grande quantidade de projeções citoplasmáticas denominadas de filapodia (Fig.6C). A avaliação da MEV ainda possibilitou verificar a
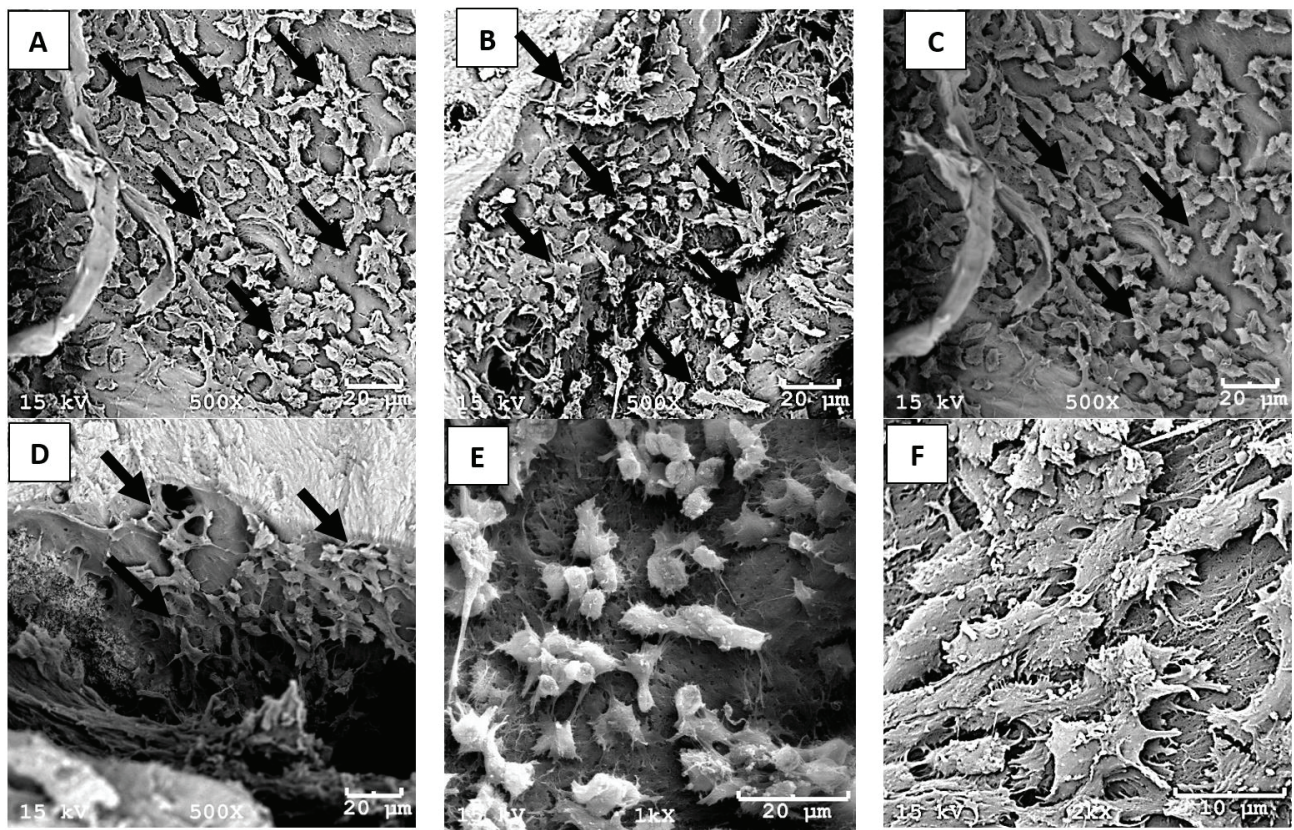

Fig.5. A microscopia eletrônica de varredura (MEV) demonstra grande quantidade de osteoblastos na superfície óssea de tíbias de ovelhas aos 3 meses de implantação. (A) Bifásico HA/TCP- $\beta$ (30/70) (setas), (B) Bifásico HA/TCP- $\beta$ (70/30) (setas), (C) TCP- $\beta$ (setas), (D) HA (setas), (E) HA/TCP- $\beta$ (80/20) e (F) HA/TCP- $\beta$ (20/80). Notar em (E) e (F) osteoblastos com grande quantidade de projeções citoplasmáticas (filapodia).
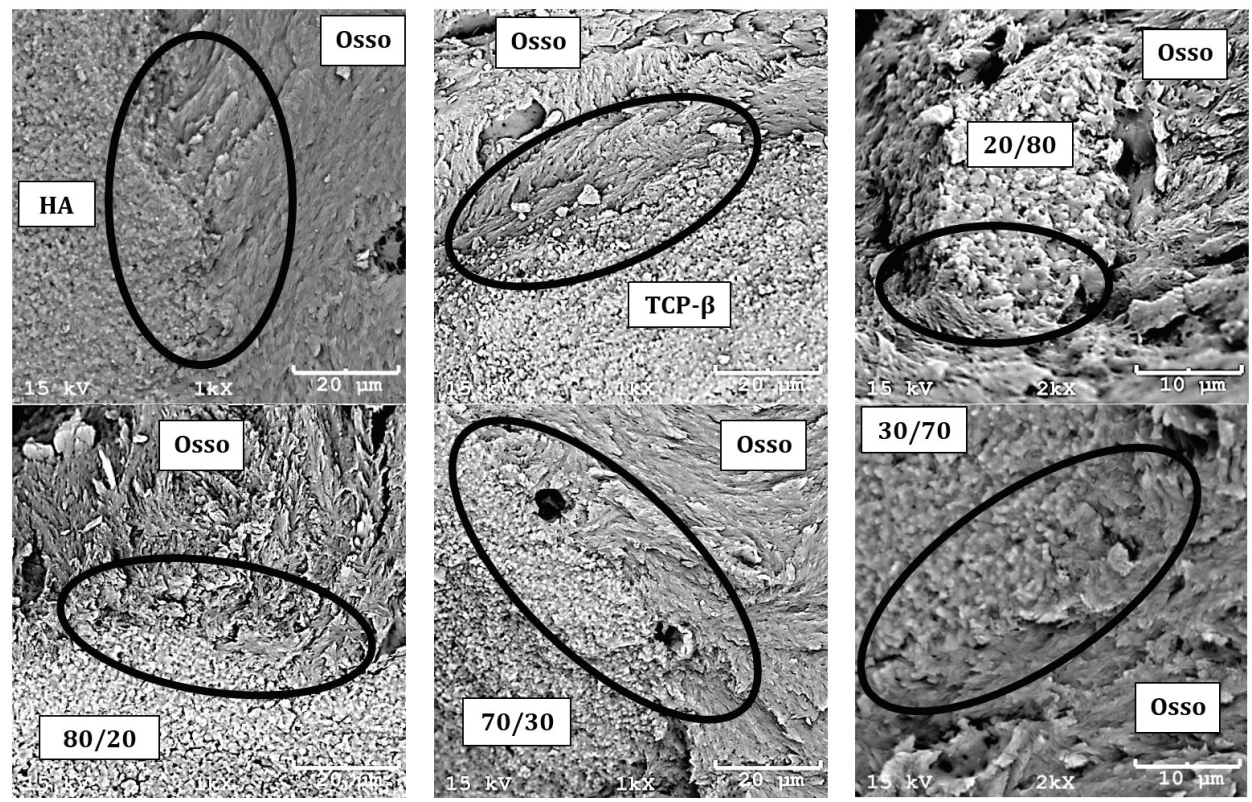

Fig.6. A microscopia eletrônica de varredura (MEV) de biomateriais enxertados em tíbias de ovelhas aos 3 meses, evidencia a interface entre o biomaterial e o osso neoformado em seis tipos de biomateriais (círculos). Notar que em todas elas existe uma estreita adesão entre osso e biomaterial. 
adesão dos biomateriais junto ao osso neoformado, em todas as amostras avaliadas foi possível verificar a justaposição na interface entre os biomateriais e o osso novo.

\section{DISCUSSÃO}

Todos os biomateriais utilizados apresentaram uma morfologia de grânulos entre 200 e $500 \mu \mathrm{m}$ e essas características são promissoras quanto à molhabilidade, capilaridade, adesão e proliferação celular na superfície de grãos e de microporos, constatação já feita por diferentes autores (Levandowski Junior 2014, Regalin 2014, Dalmônico 2015). Segundo Bohner \& Baumgart (2004), esses tamanhos de poros são classificados como de tamanho ideal para o bom desempenho das características de fixação, diferenciação e crescimento de osteoblastos e vascularização. Esses autores desenvolveram um modelo teórico da relação entre a taxa de reabsorção e tamanho do poro de fosfato de cálcio utilizado para substituição óssea. Eles chegaram à conclusão que o fosfato de cálcio microporoso impediu a penetração de células de absorção (osteoclastos e macrófacos) e de vasos sanguíneos no interior do implante. Isso também foi relatado por Mayr et al. (2015) que utilizaram TCP- $\beta$ com microporos de aproximadamente $5,39( \pm 1,33 \mu \mathrm{m})$ onde a reabsorção do biomaterial ocorreu exclusivamente em sua superfície.

O sucesso da utilização de biomateriais não depende somente da composição química do biomaterial, mas também da sua superfície e das suas características de degradação, pureza e granulometria (Van Hemert et al. 2004, Lobo \& Livingston Arinzeh 2010, Lim et al. 2015). Tendo em mente que diferentes materiais possuem diferentes características de superfície, a influência delas na reação tecidual para o acúmulo inicial de camadas de proteína pode ser alegada. As diferentes características de superfície da HA e o TCP que são causadas por disparidades nas composições químicas das superfícies e na formação dos fosfatos de cálcio, podem explicar os diferentes padrões de reações teciduais e respostas inflamatórias deles (Ghannati et al. 2012). Assim, as reações teciduais observadas em todos os grupos do presente estudo e a variação entre eles podem ser devido às diferenças nas composições químicas e na relação HA/TCP, principalmente porque estes foram os únicos parâmetros que variaram entre os diferentes biomateriais utilizados.

A remodelação óssea é um processo fisiológico firmemente ligado à absorção osteoclástica inicial, seguido de formação óssea pelos osteoblastos. A absorção osteoclástica de substituto ósseo é processo importante, uma vez que vai levar ao recrutamento de osteoblastos e substituição de enxerto ósseo por osso novo (Van Hemert et al. 2004). No presente estudo, as imagens obtidas através da MEV demonstraram a presença de osteoblastos nas superfícies ósseas de todos os biomateriais, indicando intensa atividade dessas células para todos eles. Segundo Albrektsson \& Johansson (2001), Kunert-Keil et al. (2014) e Lim et al. (2015), a presença dessas células denota característica osteocondutiva, pois possibilita que as células se fixem na superfície óssea e dos biomateriais. Essa característica é altamente desejável em um biomaterial, pois está diretamente relacionada à produção óssea. Portanto, no presente estudo, devido à presença dessas células sobre a superfície dos biomateriais, esses achados sugerem que eles apresentam grande capacidade osteocondutiva.
Lim et al. (2015) relataram em seu estudo que o bifásico HA/TCP- $\beta$ 30/70 apresentou osteoblastos com grande quantidade de filapodia dentro das partículas do biomaterial. Segundo Hong et al. (2010) a apresentação de osteoblastos com abundantes projeções citoplasmáticas (filapodia) na superfície das biocerâmicas sugere que elas têm bioatividade mais elevada, uma vez que quando a superfície é favorável para o crescimento celular, os osteoblastos podem demonstrar suas projeções. Neste estudo todos os biomateriais apresentaram grande quantidade de osteoblastos com muitas projeções citoplasmáticas (filapodia) caracterizando que eles apresentam propriedades bioativas elevadas.

Lan Levengood et al. (2010) também encontraram resultados semelhantes em seu estudo com HA, TCP- $\beta$, HA/TCP- $\beta$ 75/25 e HA/TCP- $\beta$ 25/75. Segundo esses autores o bifásico 25/75 foi o que apresentou melhor resultados dentre todos. Eles sugerem que a superfície do bifásico permite aos osteoclastos agirem de forma mais natural do que a HA ou o TCP- $\beta$ puros. Esses autores ainda sugerem que os bifásicos podem coexistir com o tecido ósseo vivo sofrendo ações naturais durante o processo de remodelação óssea.

Assim como descrito por outros autores (Van Hemert et al. 2004, Wang et al. 2007, Hong et al. 2010, Van der Pol et al. 2010, Sahithi et al. 2010, Santos et al. 2012, Saiz et al. 2013) no presente estudo os biomateriais também serviram de suporte para a formação de novo tecido ósseo, ou seja, as células foram guiadas pelo biomaterial para gerar novo tecido ósseo.

Todos os implantes estavam ligados diretamente ao osso apresentando neoformação óssea sobre as superfícies dos macroporos para todos os implantes, as células inflamatórias foram observadas muito raramente, e sua presença foi relacionado ao processo de cicatrização, assim como relatado por Theiss et al. (2005) e Von Doernberg et al. (2006).

Todos os biomateriais utilizados neste estudo apresentaram um vínculo forte e direto entre o osso e o material em todas as regiões onde foram colocados os enxertos, diferente do que encontraram Yang et al. (2014), onde essa perfeita coaptação foi encontrada especialmente na margem do defeito. Ainda segundo esses autores, a habilidade para formar osso entre os tecidos e o material, o que resulta numa interface única e forte, é uma característica especial de materiais bioativos. Dessa forma as análises de MEV do presente estudo possibilitaram verificar que todos os biomateriais remanescentes se encontraram toltalmente osteointegrados pelo osso neoformado, demonstrando a osteointegração.

0 autoenxerto resultou em rápida formação óssea e aumento da maturidade óssea aos três meses, como reportado por Jensen et al. (2007). Com o transplante de osso autólogo, ambas as células osteogênicas e moléculas osteoindutivas são trazidas até a área receptora, o que não acontece quando se utiliza materiais sintéticos como substitutivos ósseos (Jensen et al. 2007, Mayr et al. 2015).

No estudo em questão a neoformação óssea correu em paralelo com a degradação dos biomateriais, os osteoclastos foram encontrados em todos os tempos de avaliação e estas células estavam envolvidas na degradação dos biomateriais, assim como relatado por Bernstein et al. (2013) em seu estudo com TCP em articulação de joelho de ovelhas, por um período de 52 semanas.

Arinzeh et al. (2005) em seu estudo, avaliaram biomateriais em várias proporções, HA 100\%, TCP 100\%, HA/TCP 
$(76 / 24,63 / 37,56 / 44$ e $20 / 80)$, com porosidade de 60 $70 \%$ e tamanho de poros entre 300-600 $\mu \mathrm{m}$. Esses autores chegaram à conclusão que a HA e o TCP 100\% tiveram menor taxa de formação óssea (com 12 semanas), enquanto que o bifásico $20 / 80$ foi o que mostrou formação óssea mais rápida (6 semanas) e maior formação óssea no final do estudo (12 semanas), entre todos os biomateriais avaliados. Da mesma forma que encontrado pelos autores citados acima, no estudo em questão, os biomateriais que apresentaram maior absorção dos seus grânulos e substituição por osso neoformado foram os bifásicos HA/TCP- $\beta 20 / 80$ seguidos pelo HA/TCP- $\beta 30 / 70$.

No presente estudo, a avaliação das MEVs mostrou que todos os biomateriais apresentaram absorção progressiva durante o período de avaliação, no entanto, foram encontradas diferenças na quantidade de biomaterial absorvido e de osso neoformado dentro de cada grupo, nos três meses de avaliação.

Aos 3 meses, a neoformação óssea em geral aumentou em todas as amostras, com quantidade maior para os bifásicos $30 / 70$ e $20 / 80$ respectivamente. A HA e os bifásicos $80 / 20$ e 70/30 apresentaram praticamente a mesma quantidade de osso neoformado. 0 biomaterial que apresentou menor quantidade de osso neoformado foi o TCP- $\beta$ e segundo Yang et al. (2014) isto pode ser atribuído à absorção rápida de partículas de TCP- $\beta$, o que é uma característica mais dominante, do que a formação de osso, especialmente no período precoce de cicatrização.

\section{CONCLUSÕES}

As biocerâmicas sintéticas hidroxiapatita, o fosfato tricálcico- $\beta$ e os bifásicos HA/TCP- $\beta(70 / 30)$, HA/TCP- $\beta(30 / 70)$, HA/TCP- $\beta(80 / 20)$ e HA/TCP- $\beta(20 / 80)$ apresentaram boa capacidade de osteoindução, osteocondução e osteointegração, promovendo boa capacidade de estimular a formação óssea.

Na avaliação das microscopia eletrônica de varredura (MEV), foi possível observar que o bifásico HA/TCP- $\beta(20 / 80)$ foi o que apresentou maior absorção do biomaterial e formação óssea mais rápida durante a evolução da cicatrização aos 3 meses, seguido pelos biomateriais HA/TCP- $\beta(30 / 70)$, TCP- $\beta$, HA/ TCP- $\beta(70 / 30)$, HA/TCP- $\beta(80 / 20)$ e a HA, respectivamente.

\section{REFERÊNCIAS}

Albrektsson T. \& Johansson C. 2001. Osteoinduction, osteoconduction and osseointegration. Eur. Spine J. 10(Supl. 2):96-101. <http://dx.doi. org/10.1007/s005860100282><PMid:11716023>

Arinzeh T.L., Tran T., McAlary J. \& Daculsi G. 2005. A comparative study of biphasic calcium phosphate ceramics for human mesenchymal stem-cellinduced bone formation. Biomaterials 26(17):3631-3638. <http://dx.doi. org/10.1016/j.biomaterials.2004.09.035><PMid:15621253>

Bernstein A., Niemeyer P., Salzmann G., Südkamp N.P., Hube R., Klehm J., Menzel M., Von Eisenhart-Rothe R., Bohner M., Görz L. \& Mayr H.O. 2013. Microporous calcium phosphate ceramics as tissue engineering scaffolds for the repair of osteochondral defects: Histological results. Acta Biomaterialia 9(7):7490-7505. <http://dx.doi.org/10.1016/j.actbio.2013.03.021> $<$ PMid:23528497>

Bohner M. \& Baumgart F. 2004. Theoretical model to determine the effects of geometrical factors on the resorption of calcium phosphate bone substitutes. Biomaterials 25(17):3569-3582. <http://dx.doi.org/10.1016/j. biomaterials.2003.10.032><PMid:15020131>

Camargo N.H.A., Lima S.A. \& Gemelli E. 2012. Synthesis and characterization of hydroxyapatite/tio2n nanocomposites for bone tissue regeneration.
Am. J. Biomed. Engineering 2(2):41-47. <http://dx.doi.org/10.5923/j. ajbe.20120202.08>

Camargo N.H.A., Gemelli E., Moraes A.N., Costa B.D., Oleskovicz N., Dallabrida A.L. \& Lima M.P.A. 2014. Preliminary Study on Bone Neoformation Behavior of Three Types of Calcium Phosphate Bioceramics. J. Biosci. Med. 2:36-42.

CONCEA 2013. Diretriz brasileira para o cuidado e a utilização de animais para fins científicos e didáticos (DBCA). Conselho Nacional de Controle de Experimentação Animal, Ministério da Ciência, Tecnologia e Inovação, Brasília, DF. 50p.

Costa B.D., Camargo N.H.A., Oleskovicz N., Gava A., Dallabrida A.L., Regalin D., Lima M.P.A. \& Moraes A.N. 2015. Neoformação óssea e osteointegração de biomateriais micro e nanoestruturados em ovinos. Pesq. Vet. Bras. 35(2):177-187. <http://dx.doi.org/10.1590/S0100-736X2015000200015>

Costa-Rodrigues J., Fernandes A., Lopes M.A. \& Fernandes M.H. 2012. Effects of hydroxyapatite surface roughness on the differentiation of human osteoclast precursor cells. Bone 50:90. <http://dx.doi.org/10.1016/j. bone.2012.02.267>

Dalmônico G.M.L. 2011. Síntese e caracterização de fosfato de cálcio e de hidroxiapatita: elaboração de composições bifásicas HA/TCP- $\beta$ para aplicações biomédicas. Dissertação de mestrado em Ciência e Engenharia de Materiais, Universidade do Estado de Santa Catarina, Joinville, SC. 95p.

Dalmônico G.M.L. 2015. Elaboração e caracterização de biomateriais granulados microporosos de fosfatos de cálcio: teste in vivo em ovinos. Tese de Doutorado em Ciência e Engenharia de Materiais, Universidade do Estado de Santa Catarina, Joinville, SC. 202p.

Dorozhkin S.V. 2012. Biphasic, triphasic and multiphasic calcium orthophosphates. Acta Biomaterialia 8(3):963-977. <http://dx.doi. org/10.1016/j.actbio.2011.09.003><PMid:21945826>

Dubois J.C., Souchier C., Couble M.L., Exbrayat P. \& Lissac M. 1999. Na image analysis method for the study of cell adhesion to biomaterials. Biomaterials 20(19):1841-1849. <http://dx.doi.org/10.1016/S0142-9612(99)000824><PMid:10509195>

Eckstein M.B.R. 2016. Síntese e Caracterização de Pós Nanoestruturados de Fosfatos de Cálcio e Elaboração de Biomateriais. Dissertação de Mestrado em Ciência e Engenharia de Materiais, Universidade do Estado de Santa Catarina, Joinville, SC. $112 \mathrm{p}$

Flautre B., Descamps M., Delecourt C., Blary M.C. \& Hardouin P. 2001. Porous HA ceramic for bone replacement: role of the pores and interconnectionsexperimental study in the rabbit. J. Mater. Sci., Mater. Med. 12(8):679-682. <http://dx.doi.org/10.1023/A:1011256107282> <PMid:15348237>

Gauthier O., Bouler J.M., Aguado E., Pilet P. \& Daculsi G. 1998. Macroporous biphasic calcium phosphate ceramics: Influence of macropore diameter and macroporosity percentage on bone ingrowth. Biomaterials 19(1/3):133-139. <http://dx.doi.org/10.1016/S0142-9612(97)00180-4><PMid:9678860>

Ghannati S., Barbeck M., Detsch R., Deisinger U., Hilbig U., Rausch V., Sader R., Unger R.E., Ziegler G. \& Kirkpatrick C.J. 2012. The chemical composition of synthetic bone substitutes influences tissue reactions in vivo: histological and histomorphometrical analysis of the cellular inflammatory response to hydroxyapatite, beta-tricalcium phosphate and biphasic calcium phosphate ceramics. Biomed. Material 7:1-14.

Hong Y., Fan H., Li B., Guo B., Liu M. \& Zhang X. 2010. Fabrication, biological effects, and medical applications of calcium phosphates nanoceramics. Mater. Sci. Eng. Res. 70(3-6):225-242. <http://dx.doi.org/10.1016/j. mser.2010.06.010>

Jensen S.S., Yeo A., Dard M., Hunziker E., Schenk R. \& Buser D. 2007. Evaluation of a novel biphasic calcium phosphate in standardized bone defects. A histologic and histomorphometric study in the mandibles of minipigs. Clin. Oral Implants Res. 18(6):752-760. <http://dx.doi. org/10.1111/j.1600-0501.2007.01417.x><PMid:17888014>

Kunert-keil C., Scholz F., Gedrange T. \& Gredes T. 2014. Comparative study of biphasic calcium phosphate with beta-tricalcium phosphate in rat 
cranial defects: a molecular-biological and histological study. Ann. Anat. 199:79-84. <PMid:24439994>

Kurashina K., Kurita H., Wu Q., Ohtsuka A. \& Kobayashi H. 2002. Ectopic osteogenesis with biphasic ceramics of hydroxyapatite and tricalcium phosphate in rabbits. Biomaterials 23(2):407-412. <http://dx.doi. org/10.1016/S0142-9612(01)00119-3><PMid:11761160>

Lan Levengood S.K., Polak S.J., Wheeler M.B., Maki A.J., Clark S.G., Jamison R.D. \& Wagoner Johnson A.J. 2010. Multiscale osteointegration as a new paradigm for the design of calcium phosphate scaffolds for bone regeneration. Biomaterials 31(13):3552-3563. <http://dx.doi.org/10.1016/j. biomaterials.2010.01.052><PMid:20153042>

Levandowski Junior N. 2014. Análise comparativa de diferentes substitutos ósseos sintéticos microporosos em calvária de coelhos. Teses de Doutorado em Ciência e Engenharia de Materiais, Universidade do Estado de Santa Catarina, Joinville, SC. 152p.

Lim H.C., Kim K.T., Lee J.S., Jung U.W. \& Choi S.H. 2015. In vivo comparative investigation of three synthetic graft materials with varying compositions processed using different methods. Int. J. Oral Maxillofacial Implants 30(6):1280-1286. <http://dx.doi.org/10.11607/jomi.3999><PMid:26574853>

Livingston T.L., Gordon S., Archambault M., Kadiyala S., McIntosh K., Smith A. \& Peter S.J. 2003. Mesenchymal stem cells combined with biphasic calcium phosphate ceramics promote bone regeneration. J. Mater. Sci., Mater. Med. 14(3):211-218. <http://dx.doi.org/10.1023/A:1022824505404> <PMid:15348466>

Lobo S.E. \& Livingston Arinzeh T. 2010. Biphasic calcium phosphate ceramics for bone regeneration and tissue engineering applications. Material 3(2):815-826. <http://dx.doi.org/10.3390/ma3020815>

Mayr H.O., Suedkamp N.P., Hammer T., Hein W., Hube R., Roth P.V. \& Bernstein A. 2015. $\beta$-Tricalcium phosphate for bone replacement: stability and integration in sheep. J. Biomech. 48(6):1023-1031. <http://dx.doi. org/10.1016/j.jbiomech.2015.01.040><PMid:25704530>

Nuss K.M.R., Auer J.A., Boos A. \& Von Rechenberg B. 2006. An animal model in sheep for biocompatibility testing of biomaterials in cancellous bones. Biomed. Central Musculoskeletal Disorders 7(67):1-7.

Pennings E.C.M. \& Grellner W. 1989. Precise non destrutive determination of the density of ceramic. J. Am. Ceramic Soc. 72(7):1268-1270. <http:// dx.doi.org/10.1111/j.1151-2916.1989.tb09724.x>

Ramay H.R.R. \& Zhang M. 2004. Biphasic calcium phosphate nanocomposite porous scaffolds for load-bearing bone tissue engineering. Biomaterials 25(21):5171-5180. <http://dx.doi.org/10.1016/j.biomaterials.2003.12.023> $<$ PMid:15109841>

Regalin B.D.C. 2014. Comportamento da neoformação óssea e osteointegração de biomateriais micro e nano estruturados em ovinos. Dissertação de Mestrado, Centro de Ciências Agro-Veterinárias, Universidade do Estado de Santa Catarina, Lages. 86p.

Rosa A.L., Beloti M.M., Oliveira P.T. \& Van Noort R. 2002. Osseointegration and osseoconductivity of hydroxyapatite of different microporosite. J. Mater. Sci., Mater. Med.13(11):1071-1075. <http://dx.doi.org/10.1023/A:1020305008042> <PMid:15348178>
Sahithi K., Swetha M., Ramasamy K., Srinivasan N. \& Selvamurugan N. 2010. Polymeric composites containing carbon nanotubes for bone tissue engineering. Int. J. Biol. Macromolecules 46(3):281-283. <http://dx.doi. org/10.1016/j.ijbiomac.2010.01.006><PMid:20093139>

Saiz E., Zimmermann E.A., Lee J.S., Wegst U.G.K. \& Tomsia A.P. 2013. Perspectives on the role of nanotechnology in bone tissue engineering. Dental Materials 29(1):103-115. <http://dx.doi.org/10.1016/j.dental.2012.08.001> $<$ PMid:22901861>

Samavedi S., Whittington A.R. \& Goldstein A.S. 2013. Calcium phosphate ceramics in bone tissue engineering: a review of properties and their influence on cell behavior. Acta Biomaterialia 9(9):8037-8045. <http:// dx.doi.org/10.1016/j.actbio.2013.06.014><PMid:23791671>

Santos C.F.L., Silva A.P., Lopes L., Pires I. \& Correia I.J. 2012. Design and production of sintered $\beta$-tricalcium phosphate 3D scaffolds for bone tissue regeneration. Mater. Sci. Eng., C 32(5):1293-1298. <http://dx.doi. org/10.1016/j.msec.2012.04.010>

Tanaka T., Kitasato S., Chazono M., Kumagae Y., Iida T., Mitsuhashi M., Kakuta A. \& Marumo K. 2012. Use of an injeectable complex of $\beta$-tricalcium phosphate granules, hyaluronate, and fibroblast growth factor-2 on repair of unstable intertrochanteric fractures. Open Biomed. Eng. J. 6(1):98-103. <http://dx.doi.org/10.2174/1874120701206010098><PMid:23115598>

Theiss F., Apelt D., Brand B., Kutter A., Zlinszky K., Bohner M., Matter S., Frei C., Auer J.A. \& Von Rechenberg B. 2005. Biocompatibility and resorption of a new brushite calcium phosphate cement. Biomaterials 26(21):4383-4394. <http://dx.doi.org/10.1016/j.biomaterials.2004.11.056><PMid:15701367>

Van Der Pol U., Mathieu L., Zeiter S., Bourban P.E., Zambelli P.Y., Pearce S.G., Bouré L.P. \& Pioletti D.P. 2010. Augmentation of bone defect healing using a new biocomposite scaffold: an in vivo study in sheep. Acta Biomaterialia 6(9):3755-3762. <http://dx.doi.org/10.1016/j.actbio.2010.03.028> <PMid:20346421>

Van Hemert W.L.W., Willems K., Anderson P.G., Van Heerwaarden R.J. \& Wymenga A.B. 2004. Tricalcium phosphate granules or rigid wedge preforms in open wedge high tibial osteotomy: a radiological study with a new evaluation system. The Knee 11(6):451-456. <http://dx.doi. org/10.1016/j.knee.2004.08.004> <PMid:15581763>

Von Doernberg M.C., Von Rechenberg B., Bohner M., Grünenfelder S., Van Lenthe G.H., Müller R., Gasser B., Mathys R., Baroud G. \& Auer J. 2006. In vivo behavior of calcium phosphate scaffolds with four different pore sizes. Biomaterials 27(30):5186-5198. <http://dx.doi.org/10.1016/j. biomaterials.2006.05.051><PMid:16790273>

Wang H., Li Y., Zuo Y., Li J., Ma S. \& Cheng L. 2007. Biocompatibility and osteogenesis of biomimetic nanohydroxyapatite/polyamide composite scaffolds for bone tissue engineering. Biomaterials 28(22):3338-3348. <http://dx.doi.org/10.1016/j.biomaterials.2007.04.014><PMid:17481726>

Yang C., Unursaikhan O., Lee J.S., Jung U.W. \& Kim C.S. 2014. Osteoconductivity and biodegradation of synthetic bone substitutes with different tricalcium phosphate contents in rabbits. J. Biomed. Mater. Res. B, Appl Biomater. 102(1):80-88.

Yuan H., Yang Z., Li Y., Zhang X., De Bruijn J.D. \& De Groot K. 1998. Osteoinduction by calcium phosphate biomaterials. J. Mater. Sci., Mater. Med. 9(12):723726. <http://dx.doi.org/10.1023/A:1008950902047><PMid:15348929> 